\title{
Clinical Observation of Adrenaline through the Subclavian Vein Cardiac Arrest Rescue Medication
}

\author{
Yan Chen \\ People's Hospital of Zhengzhou, Zhengzhou, China
}

\begin{abstract}
Objective is to investigate the subclavian vein epinephrine rescue efficacy of the drug in patients with cardiac arrest. Methods 126 cases of cardiac arrest patients were randomly divided into the subclavian vein catheterization group and 63 cases of peripheral venous each group, and two groups were given epinephrine, with compared efficacy observation. Results: subclavian vein catheterization group of spontaneous circulation recovery time is significantly shorter than the peripheral vein group $(\mathrm{P}<0.05)$; $1 \mathrm{~h}$ recovery success rate of subclavian vein catheterization group and $24 \mathrm{~h}$ survival rates higher, the difference was statistically significant $(P<0.05)$; recovery of the amount of the drug epinephrine between the two groups, and the difference was not statistically significant $(P>0.05)$. Conclusion: To rescue cardiac arrest patients of emergency subclavian vein catheterization, intravenous accessing through the recovery of the drug can significantly shorten the recovery time of spontaneous circulation, thus improving the success rate of cardiopulmonary resuscitation early.
\end{abstract}

Keywords-Adrenaline subclavian vein under cardiac arrest

Cardiac arrest is the most critical situations often encountered in clinical practice, and adrenaline is cardiopulmonary resuscitation (CPR) during the rescue irreplaceable first-line drugs, international CPR guidelines recommend routine method of administration of epinephrine for intravenous injection $1 \mathrm{mg}$, every $3 \sim 5 \mathrm{~min}$ repeat 1 [1]. To ensure the role of adrenergic fast, we must race against time to establish smooth venous access, the opening of a peripheral vein is fast and convenient intravenous route of administration, at a time when patients are often peripheral venous puncture difficult, timeconsuming, the drug given to rescue is often some time delay, the efficacy of our hospital were randomly selected 150 cases of cardiac arrest patients cardiopulmonary resuscitation (CPR) when different routes of administration of intravenous epinephrine were compared since 2009-2012 , it is discovered in the case of the same dose of a different vein route of administration influence on the results of a large rescue, now the clinical observation of effects are summarized below.

\section{Clinical Data}

\section{A. General Information}

From 2009 to 2012, hospital emergency department patients with 150 cases of cardiac arrest were randomly divided into two groups, subclavian vein catheterization group is of 75 cases, 75 cases are of peripheral venous group, and the two groups were induced cardiac arrest primary disease, cardiac arrest time, cardioplegia type (except for advanced cardiac and respiratory cancer, wasting disease and other causes of end-stage stop) and other differences were not statistically significant $(\mathrm{P}>0.05)$, comparable.

TABLE 1 COMPARISON OF CARDIOPULMONARY RESUSCITATION IN PATIENTS WITH GENERAL INFORMATION

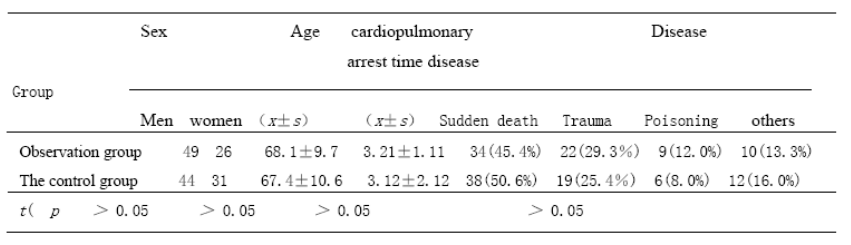

\section{B. Methods}

1.2.1 Use Beirang safety intravenous catheter with $16 \mathrm{G}$ (1.7mmx50mm, $210 \mathrm{ml} / \mathrm{min}) 1$ medals, intravenous catheter is connected $10 \mathrm{ml}$ syringe to extract adrenaline $1 \mathrm{ml}$ + saline $3 \mathrm{ml}$, supraclavicular route, puncture needle bevel up through $25-40^{\circ}$ angle $2-3 \mathrm{~cm}$ the skin around, and the needle enters the blood slowly into the syringe needle while maintaining a negative pressure, once it is into the vein, you can see the blood and smooth withdrawing the syringe, the liquid in the syringe and the upcoming return of blood push back the vein, and then there is suction, after we repeated two times unobstructed, the syringe and the needle catheter exit side while advancing the catheter jacket hose intravascular $2-3 \mathrm{~cm}$. After completely removing the needle and syringe, we connect two points after the three-way extension tubes that were connected to the infusion device and heparin cap, it is fixed with $3 \mathrm{M}$ transparent film covering the catheter jacket tube, and then we penetrate heparin hat pumping blood back after the injection of the subclavian vein every five minutes with an ordinary syringe pumping adrenaline $1.0 \mathrm{mg}$, or $2.0 \mathrm{mg}$ dose increments each 
time, until the resumption of the cycle or at doses up to $15 \mathrm{mg}(0.2 \mathrm{mg} / \mathrm{kg})$. Peripheral venous puncture method is the same as ordinary peripheral venous puncture.

1.2.2 Outcome Measures: The amount of adrenaline, restoration of spontaneous circulation time, $1 \mathrm{~h} 24 \mathrm{~h}$ recovery success rate and survival.

1.2.3 resuscitation success criteria by 2005 resuscitation guidelines criteria: (1) energy palpable arterial pulse (abdomen, neck artery), systolic blood pressure was maintained at $8 \mathrm{kPa}(60 \mathrm{~mm} \mathrm{Hg})$ above; (2) To improve peripheral circulation, lips, facial, skin, fingers turned red from pale cyanosis, limb turn temperature; (3)Apples become small and there is the emergence of light reflex; (4) recovery of spontaneous breathing; (5) Coma shallows, appears reflection, struggle or agitation.

1.2.4 Statistical Methods: Use SPSS11.5 statistical software for analysis, and measurement data using mean \pm standard deviation $(\mathrm{x} \pm \mathrm{s})$ that the groups were compared using at test, count data were compared using chi-square test, $\mathrm{P}<0.05$ was considered statistically significant.

\section{RESULTS}

Two puncture success rate was not statistically significant $(\mathrm{p}>0.05)$, the observation group set time short tube, catheter has long time compared with the control group, the difference was statistically significant $(\mathrm{P}<0.05)$, Table 2.

Recovery effect (see Table 3), differences between the two groups was statistically significant amount of adrenaline $(\mathrm{P}<0.05)$, subclavian vein catheterization group restoration of spontaneous circulation time was significantly shorter than the peripheral vein group $(\mathrm{P}<0.05)$; $1 \mathrm{~h} 24 \mathrm{~h}$ survival and recovery success rate is higher than the peripheral vein group $(\mathrm{P}<0.05)$, the difference was statistically significant.

TABle 2 Compares The Results Of Two Kinds OF Puncture Method $(\mathrm{X} \pm \mathrm{S})$

\begin{tabular}{|c|c|c|c|c|}
\hline Group & Cases & $\begin{array}{l}\text { Catheterization time } \\
(\min )\end{array}$ & (h) Catheter time & $\begin{array}{l}\text { success rate } \\
(\%)\end{array}$ \\
\hline Observer Group & 75 & $0.8 \pm 0.5$ & $12 \pm 8.1$ & $95 \%$ \\
\hline The control group & 75 & $1.4 \pm 0.6$ & $9.5 \pm 6.3$ & $91 \%$ \\
\hline$p$ & & $<0.05$ & $<0.05$ & $>0.05$ \\
\hline
\end{tabular}

TABle 3 COMPARES THE TwO GROUPS RESUSCITATION $(\mathrm{X} \pm \mathrm{S})$

\begin{tabular}{|c|c|c|c|c|c|}
\hline Goop & Cass & $\begin{array}{l}\text { The anowit of ateevilise } \\
\qquad(\mathrm{mg})\end{array}$ & 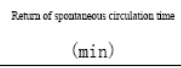 & $\begin{array}{l}\text { larreovery yxcess mate } \\
\text { (\%) }\end{array}$ & $\begin{array}{l}\text { 24t servinal } \\
(\%)\end{array}$ \\
\hline 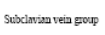 & 75 & $6.6 \pm 2.1$ & 3. $7 \pm 1.6$ & 58.7 & 21.3 \\
\hline Peripheral veב group & 75 & $12.5 \pm 4.1$ & $8.3 \pm 1.4$ & 40.0 & 10.6 \\
\hline$P$ & & $P<0.05$ & $P<0.05$ & $P<0.05$ & $P<0.05$ \\
\hline
\end{tabular}

\section{DISCUSSIONS}

3.1 To sudden cardiac arrest patients, they should be early CPR, early defibrillation and early advanced life support [2], the early clinical cardiopulmonary resuscitation measures something most commonly used which includes persistent chest cardiac massage and intravenous administration, and rescue medication Epinephrine is the drug of choice for the promotion of cardiopulmonary resuscitation, which has $\alpha$ and $\beta$ receptor stimulant dual endogenous catecholamines, where $\alpha$ receptors in the peripheral arteries are by vasoconstriction, thus reducing the capacity of the arteries, increasing the aortic diastolic pressure, thereby improving myocardial and cerebral blood supply, and it increases heart rate, myocardial contractility strengthen and promote the restoration of spontaneous circulation [3]. How to choose a reasonable route of administration during $\mathrm{CPR}$, we rescue medication adrenaline to play faster and better efficacy, it is the key to improve the success rate of research. Intravenous administration of different channels and drugs reach the central circulation time and markedly time is not the same as reported in the literature, through the center of the drug administered intravenously immediately we are able to reach the center of circulation, the 30 s were cured; and elbow administered intravenously is in order to be 1.5 a $3.0 \mathrm{~min}$ reach the central circulation, if by other smaller hand vein then it will be more time-consuming, in the case of the cycle pause from acromegaly intravenous injection, it is difficult to import the drug circulation center [4] In addition, compared with peripheral intravenous administration, intravenous drug center peak concentration is higher [5] we can see peripherally administered intravenously onset time was significantly slower than the central vein [6] we use BOLANG safety intravenous catheter needle row subclavian vein catheterization in patients with cardiac arrest rescue establish intravenous access, restoration of spontaneous circulation time, and early recovery success rate is short-term survival, it adrenalines with volume than the control group.

3.2 Deep vein puncture in the subclavian vein puncture is the most widely used, due to the relatively fixed position vein, diameter, thick, $2.0 \mathrm{~cm}$, when the patient is in the supine filling state, these characteristics determine whether it is easier to puncture success in a very short subclavian completed within the injection time, you can save valuable time and improve rescue medication speed. Peripheral vascular catheter vascular puncture selection requirements are demanding, you must select the long straight vessels, so that blood vessels in the choice's time required is extended, but also it increases the difficulty of a successful puncture; The results showed that: the subclavian vein catheterization consumes more time than the short peripheral venous puncture helps treat cardiopulmonary resuscitation. Therefore, if emergency personnel have sufficient experience, and are skilled in operation and accurate, they select the subclavian vein catheterization as the ideal deep vein to puncture a vein [7].

3.3 Indwelling venipuncture needle subclavian administered cardiopulmonary arrest patients rescued is safe operation, the method is simple; puncture and supraclavicular route can be done without termination under endotracheal intubation, such as chest compressions and rescue operation has been completed, so that the drug can rescue rapidly into the central circulation, and it plays a 
important role in the rapid rescue of patients to gain time, it is an ideal method for the recovery of the patient pathway and emergency medication.

Combined with the above advantages, the present study was designed in two veins and peripheral venous subclavian vein is contrasted to the adrenaline effect of cardiopulmonary resuscitation. Our results show that: the rescue cardiopulmonary arrest, perform CPR in an emergency during the subclavian vein catheterization, and intravenous channel through which can be quickly restored to the recovery of spontaneous circulation, it significantly improves the survival rate of patients with foreign literature [9] studies which have reported the same. Under the proposal that does not interfere when CPR chest compressions circumstances, it can be considered by those who have experienced the rapid establishment of intravenous access rescue injection drug recovery subclavian to improve survival.

\section{REFERENCES}

[1] Chenguowei. Within the heart of modern science [M]. Changsha: Hunan Science and Technology Press, 1995: 525-526.
[2] Fang Xiangshao, Fu Yue, Huangzi Tong. Interpretation of the "2005 International Cardiopulmonary Resuscitation and Emergency Cardiovascular Care Guide" - basic life support Lingnan Journal of Emergency Medicine, 2006,11 (3): 239-241.

[3] Wang Jingying. Discussion on cardiopulmonary resuscitation when the best mode of administration of epinephrine hydrochloride [J] contemporary medicine, 2010,16 (7): 10.

[4] Yang Hui, Gao Jianwei. Subclavian vein puncture in the emergency rescue and care in clinical application [J] Chinese Medicine, 2009,6 (25): 86-87.

[5] Li Chun-Sheng. Principles of modern resuscitation drug application should have [J] Chinese Critical Care Medicine, 2000,12 (11): 643644

[6] Wu Jinbo, WANG Zhi-bing, Hu Yue-hong, et al. Clinical application of Emergency deep venous catheterization during cardiopulmonary resuscitation [J] .2013,14 (4): 173-174.

[7] Zhang Bangqun, Zhai Cheng Shun. Subclavian vein catheterization in critically ill patients in the emergency rescue application [J] Medical Information (in Xunkan), 2010, (08): 2084-2085.

[8] Redding MI, Greenberg PC The use of endot reacheal medication in cardiac emergencies [J] Resus Citation, 1994, 22 (2): 155. 